\title{
Contributions of the fixed point technique to solve the 2D Volterra integral equations, Riemann-Liouville fractional integrals, and Atangana-Baleanu integral operators
}

\author{
Hasanen A. Hammad ${ }^{1}$ (D) Hassen Aydi $^{2,3,4^{*}}$ (D) and Nabil Mlaiki ${ }^{5}$ (D)
}

\author{
"Correspondence: \\ hassen.aydi@isima.rnu.tn \\ ${ }^{2}$ Institut Supérieur d'Informatique et \\ des Techniques de Communication, \\ Université de Sousse, $H$. Sousse, \\ Tunisia \\ ${ }^{3}$ Department of Mathematics and \\ Applied Mathematics, Sefako \\ Makgatho Health Sciences \\ University, Ga-Rankuwa, South \\ Africa \\ Full list of author information is \\ available at the end of the article
}

\begin{abstract}
In this manuscript, some fixed point results for generalized contractive type mappings under mild conditions in the setting of double controlled metric spaces (in short, $\eta_{\text {- }}$-metric spaces) are obtained. Moreover, some related consequences dealing with a common fixed point concept and nontrivial examples to support our results are presented. Ultimately, we use the theoretical results to discuss the existence and uniqueness of solutions of 2D Volterra integral equations, Riemann-Liouville integrals and Atangana-Baleanu integral operators are given.
\end{abstract}

MSC: 26A33; 34A08; 34B24; 39A70; 47H10; 54H25

Keywords: Double controlled metric spaces; 2D Volterra integral equations; Riemann-Liouville fractional integrals; Atangana-Baleanu integral operators

\section{Introduction}

Fixed point theorems have numerous applications in mathematics and are applied in diverse fields as biology [1], chemistry [2], economics [3, 4], engineering [5], game theory [6], and physics [7, 8]. In the last years, boundary value problems of nonlinear fractional differential equations with a variety of boundary conditions have been studied by various researchers, see [9-20]. Fractional differential equations appear naturally in diverse fields of science and engineering. They constitute an important field of research. It should be noted that most papers dealing with the existence of solutions of nonlinear initial value problems of fractional differential equations mainly use the techniques of nonlinear analysis such as fixed point techniques, stability, the Leray-Schauder result, etc. Relatively, fractional calculus and fractional differential/integral equations are very fresh topics for the researchers. For instance, in [21] the authors resolved some fractional differential equations with multiple delays in relation to chaos neuron models by using fixed point results of Lou [22] and E. de Pascale and L. de Pascale [23]. Amann [24] used a fixed point technique when studying some nonlinear eigenvalue problems in ordered Banach spaces. Liu et al. [25] gave applications of mixed monotone operators with superlinear nonlinearity

(c) The Author(s) 2021. This article is licensed under a Creative Commons Attribution 4.0 International License, which permits use sharing, adaptation, distribution and reproduction in any medium or format, as long as you give appropriate credit to the original author(s) and the source, provide a link to the Creative Commons licence, and indicate if changes were made. The images or other third party material in this article are included in the article's Creative Commons licence, unless indicated otherwise in a credit line to the material. If material is not included in the article's Creative Commons licence and your intended use is not permitted by statutory regulation or exceeds the permitted use, you will need to obtain permission directly from the copyright holder. To view a copy of this licence, visit http://creativecommons.org/licenses/by/4.0/. 
via fixed point theory. On the other hand, by using fixed point theorems, the existence and uniqueness of solutions to differential/integral equations involving fractional operators were studied by a huge number of researchers. For further related results, see for example [26-30].

Definition 1.1 ([31]) The usual form of the RL-fractional integral operator of order $\tau$ is

$$
I^{\tau} \partial(\alpha)=\frac{1}{\Gamma(\tau)} \int_{0}^{\alpha}(\alpha-\hbar)^{\tau-1} \partial(\hbar) d \hbar
$$

where $\tau>0$, and the function $\partial$ is defined on $L^{1}\left(\mathbb{R}^{+}\right)$.

Definition 1.2 ([31]) Let $\partial:(0, \infty) \rightarrow \mathbb{R}$ be a continuous function, the RL-fractional derivative of order $\tau$ is defined as

$$
D^{\tau} \partial(\alpha)=\frac{1}{\Gamma(\eta-\tau)}\left(\frac{d}{d \alpha}\right)^{n} \int_{0}^{\alpha}(\alpha-\hbar)^{\eta-\tau-1} \partial(\hbar) d \hbar,
$$

where $n=[\tau]+1$.

In this manuscript, we investigate some fixed point results via a class of contractive type mappings involving mild conditions in the setting of $\eta_{\beth}^{v}$-metric spaces. Also, some nontrivial examples are introduced. Finally, as applications, theoretical results are involved to discuss the existence and uniqueness of a solution of 2D Volterra integral equations, Riemann-Liouville integrals, and Atangana-Baleanu integral operators.

\section{Main results}

We begin this section with the following definition.

Definition 2.1 Suppose that $\wp$ is a nonempty set and $J, v: \wp \times \wp \rightarrow[1, \infty)$ are given functions. Let $\eta_{\beth}^{\nu}: \wp \times \wp \rightarrow[0, \infty)$ be a distance function on $\wp$. We list the following hypotheses for all $\varsigma, v, \tau \in \wp$ :

(J) $\eta_{\beth}^{v}(\varsigma, v)=0$ iff $\varsigma=v$;

$\left(J_{2}\right) \eta_{\beth}^{v}(\varsigma, v)=\eta_{\beth}^{v}(v, \varsigma)$

(J) $\eta_{\beth}^{\nu}(\varsigma, v) \leq \Xi(\varsigma, v)\left[\eta_{\beth}^{\nu}(\varsigma, \tau)+\eta_{\beth}^{\nu}(\tau, v)\right]$ (an extended triangle inequality);

$\left(J_{4}\right) \eta_{\beth}^{\nu}(\varsigma, v) \leq \Xi(\varsigma, \tau) \eta_{\beth}^{\nu}(\varsigma, \tau)+\Xi(\tau, v) \eta_{\beth}^{\nu}(\tau, v)$ (a controlled triangle inequality);

(J5) $\eta_{\beth}^{v}(\varsigma, v) \leq \Xi(\varsigma, \tau) \eta_{\beth}^{v}(\varsigma, \tau)+v(\tau, v) \eta_{\beth}^{v}(\tau, v)$ (a double controlled triangle inequality).

$\eta_{\beth}^{v}$ is called:

- extended $b$ - [32] if $\eta_{\beth}^{v}$ satisfies $\left(J_{1}\right)-\left(J_{3}\right)$;

- controlled metric type [33] if $\eta_{\beth}^{\nu}$ satisfies $\left(J_{1}\right),\left(J_{2}\right)$, and $\left(J_{4}\right)$;

- double controlled metric type [34] if $\eta_{\beth}^{v}$ satisfies $\left(J_{1}\right),\left(J_{2}\right)$, and $\left(J_{5}\right)$.

The pair $\left(\wp, \eta_{\beth}^{\nu}\right)$ is called an extended $b$-metric/ a controlled metric type/ a double controlled metric type space if $\eta_{\beth}^{v}$ is extended $b$-metric/ a controlled metric type/ a double controlled metric type on $\wp$, respectively.

Example 2.2 Let $\wp=\{1,2,3\}$. Define $], v: \wp \times \wp \rightarrow[1, \infty)$ by

$$
I(1,2)=I(2,1)=1, \quad I(2,3)=I(3,2)=\frac{8}{7}, \quad \text { and } \quad I(1,3)=I(3,1)=\frac{3}{2},
$$




$$
v(1,2)=v(2,1)=\frac{7}{6}, \quad v(2,3)=v(3,2)=\frac{9}{2}, \quad \text { and } \quad v(1,3)=v(3,1)=2 .
$$

Let $\eta_{\beth}^{v}: \wp \times \wp \rightarrow[0, \infty)$ be given as

$$
\begin{array}{ll}
\eta_{\beth}^{\nu}(1,3)=\eta_{\beth}^{\nu}(3,1)=1, & \eta_{\beth}^{\nu}(1,2)=\eta_{\beth}^{\nu}(2,1)=2, \\
\eta_{\beth}^{v}(2,3)=\eta_{\beth}^{v}(3,2)=3.6, & \eta_{\beth}^{v}(1,1)=\eta_{\beth}^{v}(2,2)=\eta_{\beth}^{v}(3,3)=0 .
\end{array}
$$

Note that

$$
\begin{aligned}
& \eta_{\beth}^{v}(2,3) \geq \Xi(2,3)\left[\eta_{\beth}^{v}(2,1)+\eta_{\beth}^{\nu}(1,3)\right], \\
& \eta_{\beth}^{v}(2,3) \geq \Xi(2,1) \eta_{\beth}^{v}(2,1)+\beth(1,3) \eta_{\beth}^{v}(1,3),
\end{aligned}
$$

and

$$
\begin{aligned}
& \eta_{\beth}^{\nu}(2,3) \leq \beth(2,1) \eta_{\beth}^{\nu}(2,1)+v(1,3) \eta_{\beth}^{\nu}(1,3), \\
& \eta_{\beth}^{\nu}(1,2) \leq \beth(1,3) \eta_{\beth}^{\nu}(1,3)+v(3,2) \eta_{\beth}^{v}(3,2) \text {, } \\
& \eta_{\beth}^{\nu}(1,3) \leq \beth(1,2) \eta_{\beth}^{\nu}(1,2)+v(2,3) \eta_{\beth}^{\nu}(2,3) .
\end{aligned}
$$

Thus, $\eta_{\beth}^{v}$ is neither extended $b$-metric nor controlled metric type for the function I, but it is double controlled metric type.

Definition 2.3 Let $\left(\wp, \eta_{\beth}^{\nu}\right)$ be a double controlled metric type space (for short, $\eta_{\beth}^{\nu}$-metric space). A sequence $\left\{\varsigma_{n}\right\} \in \wp$ is called:

- convergent if there is $\varsigma \in \wp$ such that $\lim _{n \rightarrow \infty} \eta_{\beth}^{v}\left(\varsigma_{n}, \varsigma\right)=0$, and this notation leads to $\lim _{n \rightarrow \infty} \varsigma_{n}=\varsigma$;

- Cauchy iff $\eta_{\beth}^{v}\left(\varsigma_{n}, \varsigma_{m}\right) \rightarrow 0$ as $n, m \rightarrow \infty$.

An $\eta_{\beth}^{v}$-metric space is complete if every Cauchy sequence in $\wp$ is convergent.

Remark 2.4 If $I(\zeta, \tau)=v(\tau, v)=b \geq 1$ for all $\zeta, v, \tau \in \wp$, then an $\eta_{\beth}^{v}$-metric space reduces to a $b$-metric space $[35,36]$.

Theorem 2.5 Let $\left(\wp, \eta_{\beth}^{\nu}\right)$ be a complete $\eta_{\beth}^{\nu}$-metric space and the mappings $\partial, \mho: \wp \rightarrow \wp$ satisfy

$$
\begin{aligned}
a^{b} \eta_{\beth}^{v}(\partial \varsigma, \mho v) \leq & \sigma \eta_{\beth}^{v}(\varsigma, v)+\rho \frac{\eta_{\beth}^{v}(v, \mho v)\left[1+\eta_{\beth}^{v}(v, \mho v)\right]}{1+\eta_{\beth}^{v}(\partial \varsigma, \mho v)} \\
& +\mu \frac{\eta_{\beth}^{v}(v, \mho v)\left[1+\eta_{\beth}^{v}(\varsigma, \partial \varsigma)\right]}{1+\eta_{\beth}^{v}(\varsigma, v)}
\end{aligned}
$$

for all $\varsigma, v \in \wp$, where $\sigma, \rho$, and $\mu$ are nonnegative real numbers with $\sigma+\rho+\mu<1$ and $a, b \geq 1$. Consider $\varsigma_{n}=\mho^{n} \varsigma_{\circ}$ for $\zeta_{\circ} \in \wp$. Then $\partial$ and $\mho$ have a unique common fixed point (cfp), provided that the following hypotheses hold:

(i)

$$
\sup _{l \geq 1} \lim _{j \rightarrow \infty} \frac{I\left(\varsigma_{j+1}, \varsigma_{j+2}\right)}{I\left(\varsigma_{j}, \varsigma_{j+1}\right)} v\left(\varsigma_{j+1}, \varsigma_{l}\right)<\frac{1}{\Theta}, \quad \text { where } \Theta=\frac{\sigma}{a^{b}-\rho-\mu}
$$


(ii) $\lim _{n \rightarrow \infty} I\left(\varsigma, \varsigma_{n}\right)$ and $\lim _{n \rightarrow \infty} v\left(\varsigma, \varsigma_{n}\right)$ exist and are finite for all $\varsigma \in \wp$.

Proof Suppose that $\varsigma_{0} \in \wp$ is an arbitrary point such that the sequence $\left\{\varsigma_{n}=\mho^{n} \varsigma_{0}\right\}$ in $\wp$ satisfies the two conditions of theorem and iterates below as follows:

$$
\varsigma_{2 n+1}=\partial \varsigma_{2 n} \quad \text { and } \quad \varsigma_{2 n+2}=\mho_{\varsigma_{2 n+1}}, \quad n=0,1, \ldots
$$

From inequality (2.1), one writes

$$
\begin{aligned}
& a^{b} \eta_{\beth}^{v}\left(\varsigma_{2 n+1}, \varsigma_{2 n+2}\right)=a^{b} \eta_{\beth}^{v}\left(\partial \varsigma_{2 n}, \mho \varsigma_{2 n+1}\right) \\
& \leq \sigma \eta_{\beth}^{v}\left(\varsigma_{2 n}, \varsigma_{2 n+1}\right)+\rho \frac{\eta_{\beth}^{v}\left(\varsigma_{2 n+1}, \mho_{\varsigma_{2 n+1}}\right)\left[1+\eta_{\beth}^{v}\left(\varsigma_{2 n+1}, \mho_{\varsigma_{2 n+1}}\right)\right]}{1+\eta_{\beth}^{v}\left(\partial \varsigma_{2 n}, \mho_{\varsigma_{2 n+1}}\right)} \\
& +\mu \frac{\eta_{\beth}^{\nu}\left(\varsigma_{2 n+1}, \mho \varsigma_{2 n+1}\right)\left[1+\eta_{\beth}^{\nu}\left(\varsigma_{2 n}, \partial \varsigma_{2 n}\right)\right]}{1+\eta_{\beth}^{\nu}\left(\varsigma_{2 n}, \varsigma_{2 n+1}\right)} \\
& =\sigma \eta_{\beth}^{\nu}\left(\varsigma_{2 n}, \varsigma_{2 n+1}\right)+\rho \frac{\eta_{\beth}^{\nu}\left(\varsigma_{2 n+1}, \varsigma_{2 n+2}\right)\left[1+\eta_{\beth}^{\nu}\left(\varsigma_{2 n+1}, \varsigma_{2 n+2}\right)\right]}{1+\eta_{\beth}^{\nu}\left(\varsigma_{2 n+1}, \mho_{\varsigma_{2 n+2}}\right)} \\
& +\mu \frac{\eta_{\beth}^{\nu}\left(\varsigma_{2 n+1}, \varsigma_{2 n+2}\right)\left[1+\eta_{\beth}^{v}\left(\varsigma_{2 n}, \varsigma_{2 n+1}\right)\right]}{1+\eta_{\beth}^{v}\left(\varsigma_{2 n}, \varsigma_{2 n+1}\right)} \\
& =\sigma \eta_{\beth}^{\nu}\left(\varsigma_{2 n}, \varsigma_{2 n+1}\right)+(\rho+\mu) \eta_{\beth}^{\nu}\left(\varsigma_{2 n+1}, \varsigma_{2 n+2}\right) \text {. }
\end{aligned}
$$

This leads to

$$
\eta_{\beth}^{\nu}\left(\varsigma_{2 n+1}, \varsigma_{2 n+2}\right) \leq\left(\frac{\sigma}{a^{b}-\rho-\mu}\right) \eta_{\beth}^{\nu}\left(\varsigma_{2 n}, \varsigma_{2 n+1}\right) .
$$

Iteratively,

$$
\eta_{\beth}^{\nu}\left(\varsigma_{2 n+2}, \varsigma_{2 n+3}\right) \leq\left(\frac{\sigma}{a^{b}-\rho-\mu}\right) \eta_{\beth}^{\nu}\left(\varsigma_{2 n+1}, \varsigma_{2 n+2}\right) .
$$

By taking $\Theta=\frac{\sigma}{a^{b}-\rho-\mu}<1$, one gets

$$
\begin{aligned}
\eta_{\beth}^{\nu}\left(\varsigma_{n}, \varsigma_{n+1}\right) \leq & \Theta \eta_{\beth}^{\nu}\left(\varsigma_{n-1}, \varsigma_{n}\right) \\
\leq & \Theta^{2} \eta_{\beth}^{v}\left(\varsigma_{n-2}, \varsigma_{n-1}\right) \\
& \vdots \\
\leq & \Theta^{n} \eta_{\beth}^{v}\left(\varsigma_{0}, \varsigma_{1}\right) .
\end{aligned}
$$

For all $m, n \in \mathbb{N}$ with $m>n$, we get

$$
\begin{aligned}
& \eta_{\beth}^{v}\left(\varsigma_{n}, \varsigma_{m}\right) \leq I\left(\varsigma_{n}, \varsigma_{n+1}\right) \eta_{\beth}^{v}\left(\varsigma_{n}, \varsigma_{n+1}\right)+v\left(\varsigma_{n+1}, \varsigma_{m}\right) \eta_{\beth}^{v}\left(\varsigma_{n+1}, \varsigma_{m}\right) \\
& \leq I\left(\varsigma_{n}, \varsigma_{n+1}\right) \eta_{\beth}^{v}\left(\varsigma_{n}, \varsigma_{n+1}\right)+v\left(\varsigma_{n+1}, \varsigma_{m}\right)\left[\begin{array}{c}
J\left(\varsigma_{n+1}, \varsigma_{n+2}\right) \eta_{\beth}^{v}\left(\varsigma_{n+1}, \varsigma_{n+2}\right) \\
+v\left(\varsigma_{n+2}, \varsigma_{m}\right) \eta_{\beth}^{v}\left(\varsigma_{n+2}, \varsigma_{m}\right)
\end{array}\right] \\
&=I\left(\varsigma_{n}, \varsigma_{n+1}\right) \eta_{\beth}^{v}\left(\varsigma_{n}, \varsigma_{n+1}\right)+v\left(\varsigma_{n+1}, \varsigma_{m}\right) \beth\left(\varsigma_{n+1}, \varsigma_{n+2}\right) \eta_{\beth}^{v}\left(\varsigma_{n+1}, \varsigma_{n+2}\right) \\
&+v\left(\varsigma_{n+1}, \varsigma_{m}\right) v\left(\varsigma_{n+2}, \varsigma_{m}\right) \eta_{\beth}^{v}\left(\varsigma_{n+2}, \varsigma_{m}\right)
\end{aligned}
$$




$$
\begin{aligned}
& \leq \beth\left(\varsigma_{n}, \varsigma_{n+1}\right) \eta_{\beth}^{\nu}\left(\varsigma_{n}, \varsigma_{n+1}\right)+\sum_{i=n+1}^{m-2}\left(\prod_{j=n+1}^{i} v\left(\varsigma_{j}, \varsigma_{m}\right)\right) J\left(\varsigma_{i}, \varsigma_{i+1}\right) \eta_{\beth}^{\nu}\left(\varsigma_{i}, \varsigma_{i+1}\right) \\
& +\prod_{k=n+1}^{m-1} v\left(\varsigma_{k}, \varsigma_{m}\right) \eta_{\beth}^{v}\left(\varsigma_{m-1}, \varsigma_{m}\right) \\
& \leq \beth\left(\varsigma_{n}, \varsigma_{n+1}\right) \Theta^{n} \eta_{\beth}^{\nu}\left(\varsigma_{0}, \varsigma_{1}\right)+\sum_{i=n+1}^{m-2}\left(\prod_{j=n+1}^{i} v\left(\varsigma_{j}, \varsigma_{m}\right)\right) J\left(\varsigma_{i}, \varsigma_{i+1}\right) \Theta^{i} \eta_{\beth}^{v}\left(\varsigma_{0}, \varsigma_{1}\right) \\
& +\prod_{k=n+1}^{m-1} v\left(\varsigma_{k}, \varsigma_{m}\right) \Theta^{m-1} \eta_{\beth}^{\nu}\left(\varsigma_{0}, \varsigma_{1}\right) \\
& \leq \beth\left(\varsigma_{n}, \varsigma_{n+1}\right) \Theta^{n} \eta_{\beth}^{\nu}\left(\varsigma_{0}, \varsigma_{1}\right)+\sum_{i=n+1}^{m-2}\left(\prod_{j=n+1}^{i} v\left(\varsigma_{j}, \varsigma_{m}\right)\right) J\left(\varsigma_{i}, \varsigma_{i+1}\right) \Theta^{i} \eta_{\beth}^{v}\left(\varsigma_{0}, \varsigma_{1}\right) \\
& \left.+\prod_{k=n+1}^{m-1} v\left(\varsigma_{k}, \varsigma_{m}\right) \Theta^{m-1}\right\rfloor\left(\varsigma_{m-1}, \varsigma_{m}\right) \eta_{\beth}^{v}\left(\varsigma_{0}, \varsigma_{1}\right) \\
& \leq \beth\left(\varsigma_{n}, \varsigma_{n+1}\right) \Theta^{n} \eta_{\beth}^{\nu}\left(\varsigma_{0}, \varsigma_{1}\right)+\sum_{i=n+1}^{m-1}\left(\prod_{j=n+1}^{i} v\left(\varsigma_{j}, \varsigma_{m}\right)\right) J\left(\varsigma_{i}, \varsigma_{i+1}\right) \Theta^{i} \eta_{\beth}^{v}\left(\varsigma_{0}, \varsigma_{1}\right) \\
& \leq \beth\left(\varsigma_{n}, \varsigma_{n+1}\right) \Theta^{n} \eta_{\beth}^{\nu}\left(\varsigma_{0}, \varsigma_{1}\right)+\sum_{i=n+1}^{m-1}\left(\prod_{j=0}^{i} v\left(\varsigma_{j}, \varsigma_{m}\right)\right) J\left(\varsigma_{i}, \varsigma_{i+1}\right) \Theta^{i} \eta_{\beth}^{\nu}\left(\varsigma_{0}, \varsigma_{1}\right) .
\end{aligned}
$$

Set $\Lambda_{\ell}=\sum_{i=0}^{\ell}\left(\prod_{j=0}^{i} v\left(\varsigma_{j}, \varsigma_{m}\right)\right) \beth\left(\varsigma_{i}, \varsigma_{i+1}\right) \Theta^{i}$. Hence, inequality (2.4) implies that

$$
\eta_{\beth}^{\nu}\left(\varsigma_{n}, \varsigma_{m}\right) \leq \eta_{\beth}^{\nu}\left(\varsigma_{0}, \varsigma_{1}\right)\left[\Theta^{n} \beth\left(\varsigma_{n}, \varsigma_{n+1}\right)+\left(\Lambda_{m-1}-\Lambda_{n}\right)\right] .
$$

It follows by (2.2) and the ratio test that the real number sequence $\left\{\Lambda_{n}\right\}$ exists, and so $\left\{\Lambda_{n}\right\}$ is Cauchy. Note that the ratio test is applied to the term $\varkappa_{i}=\left(\prod_{j=0}^{i} v\left(\varsigma_{j}, \varsigma_{m}\right)\right) I\left(\varsigma_{i}, \varsigma_{i+1}\right)$. Taking $n, m \rightarrow \infty$ in (2.5), we can write

$$
\lim _{n, m \rightarrow \infty} \eta_{\beth}^{v}\left(\varsigma_{n}, \varsigma_{m}\right)=0
$$

Relation (2.6) implies that the sequence $\left\{\zeta_{n}\right\}$ is Cauchy. The completeness of $\left(\wp, \eta_{\beth}^{v}\right)$ yields that there exists some $\varrho \in \wp$ such that

$$
\lim _{n \rightarrow \infty} \eta_{\beth}^{\nu}\left(\varsigma_{n}, \varrho\right)=0
$$

Applying the triangle inequality, we have

$$
\eta_{\beth}^{\nu}\left(\varrho, \varsigma_{n+1}\right) \leq \beth\left(\varrho, \varsigma_{n}\right) \eta_{\beth}^{\nu}\left(\varrho, \varsigma_{n}\right)+v\left(\varsigma_{n}, \varsigma_{n+1}\right) \eta_{\beth}^{\nu}\left(\varsigma_{n}, \varsigma_{n+1}\right) .
$$

By (2.6) and (2.7) in (2.8), we get at the limit

$$
\lim _{n \rightarrow \infty} \eta_{\beth}^{\nu}\left(\varrho, \varsigma_{n+1}\right)=0 .
$$


Now, we shall show that $\varrho=\partial \varrho$. Using the method of contradiction, i.e., let $\eta_{\beth}^{v}(\varrho, \partial \varrho)>0$.

Then

$$
\begin{aligned}
& \eta_{\beth}^{\nu}(\varrho, \partial \varrho) \leq \beth\left(\varrho, \varsigma_{n+2}\right) \eta_{\beth}^{\nu}\left(\varrho, \varsigma_{n+2}\right)+v\left(\varsigma_{n+2}, \partial \varrho\right) \eta_{\beth}^{\nu}\left(\varsigma_{n+2}, \partial \varrho\right) \\
& =\beth\left(\varrho, \varsigma_{n+2}\right) \eta_{\beth}^{\nu}\left(\varrho, \varsigma_{n+2}\right)+v\left(\varsigma_{n+2}, \partial \varrho\right) \eta_{\beth}^{v}\left(\mho \varsigma_{n+1}, \partial \varrho\right) \\
& \leq \beth\left(\varrho, \varsigma_{n+2}\right) \eta_{\beth}^{\nu}\left(\varrho, \varsigma_{n+2}\right)+v\left(\varsigma_{n+2}, \partial \varrho\right) a^{b} \eta_{\beth}^{\nu}\left(\partial \varrho, \mho \varsigma_{n+1}\right)
\end{aligned}
$$

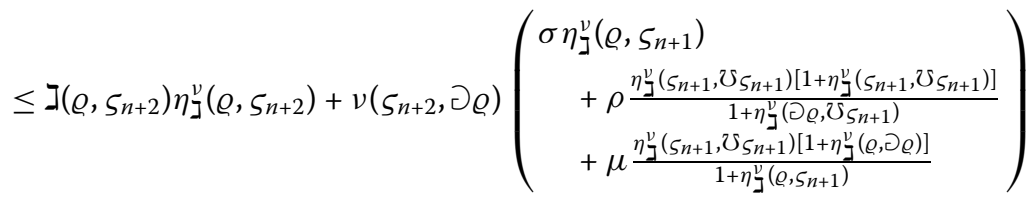

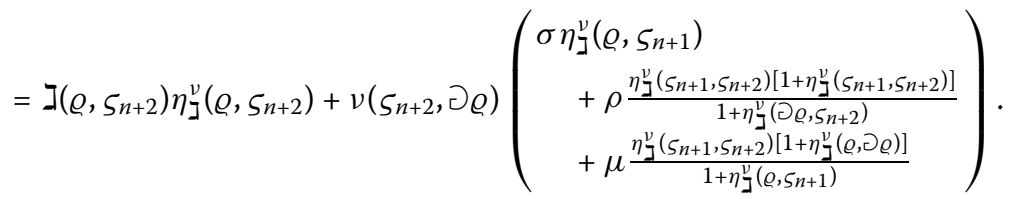

Letting $n \rightarrow \infty$ in the above inequality and with the help of (2.6), (2.7), and (2.9), we conclude that $\eta_{\beth}^{\nu}(\varrho, \partial \varrho)=0$. It is a contradiction, that is, $\varrho=\partial \varrho$. Likewise, we can show that $\varrho=\mho \varrho$. This means that $\varrho$ is a $\operatorname{cfp}$ of $\circlearrowright$ and $\mho$. For uniqueness, suppose that $\alpha$ is another $\operatorname{cfp}$ of $\partial$ and $\mho$ such that $\varrho \neq \alpha$, then by condition (2.1) we obtain that

$$
\begin{aligned}
a^{b} \eta_{\beth}^{\nu}(\varrho, \alpha) & =a^{b} \eta_{\beth}^{\nu}(\partial \varrho, \mho \alpha) \\
& \leq \sigma \eta_{\beth}^{\nu}(\varrho, \alpha)+\rho \frac{\eta_{\beth}^{\nu}(\alpha, \mho \alpha)\left[1+\eta_{\beth}^{\nu}(\alpha, \mho \alpha)\right]}{1+\eta_{\beth}^{\nu}(\partial \varrho, \mho \alpha)}+\mu \frac{\eta_{\beth}^{\nu}(\alpha, \mho \alpha)\left[1+\eta_{\beth}^{\nu}(\varrho, \partial \varrho)\right]}{1+\eta_{\beth}^{\nu}(\varrho, \alpha)} \\
& =\sigma \eta_{\beth}^{\nu}(\varrho, \alpha) .
\end{aligned}
$$

This implies that $\left(a^{b}-\sigma\right) \eta_{\beth}^{v}(\varrho, \alpha) \leq 0$, a contradiction. So it should be $\eta_{\beth}^{v}(\mu, \alpha)=0$, i.e., $\mu=\alpha$. Hence $\mu$ is the unique cfp of $\supset$ and $\mho$. This ends the proof.

Theorem 2.5 reduces to the following corollary if we consider that the two mappings $\partial$ and $\mho$ are equal.

Corollary 2.6 Let $\left(\wp, \eta_{\beth}^{\nu}\right)$ be a complete $\eta_{\beth}^{\nu}$-metric space and $\partial: \wp \rightarrow \wp$ be a mapping satisfying

$$
a^{b} \eta_{\beth}^{v}(\partial \varsigma, \partial v) \leq \sigma \eta_{\beth}^{v}(\varsigma, v)+\rho \frac{\eta_{\beth}^{\nu}(v, \partial v)\left[1+\eta_{\beth}^{\nu}(v, \partial v)\right]}{1+\eta_{\beth}^{v}(\partial \varsigma, \partial v)}+\mu \frac{\eta_{\beth}^{\nu}(v, \partial v)\left[1+\eta_{\beth}^{\nu}(\varsigma, \partial \varsigma)\right]}{1+\eta_{\beth}^{v}(\varsigma, v)}
$$

for all $\varsigma, v \in \wp$, where $\sigma, \rho$, and $\mu$ are nonnegative real numbers with $\sigma+\rho+\mu<1$ and $a, b \geq 1$. Consider $\varsigma_{n}=\partial^{n} \varsigma_{\circ}$ for $\zeta_{\circ} \in \wp$. If assumptions ( $i$ ) and (ii) of Theorem 2.5 are fulfilled, then $\partial$ has a unique fixed point.

If we take $a=b=1, \mu=\rho=0$, and $\partial=\mho$ in Theorem 2.5, we get the following main result. 
Corollary 2.7 Let $\left(\wp, \eta_{\beth}^{v}\right)$ be a complete $\eta_{\beth}^{v}$-metric space, and let the mapping $\partial: \wp \rightarrow \wp$ satisfy

$$
\eta_{\beth}^{v}(\partial \varsigma, \partial v) \leq \sigma \eta_{\beth}^{v}(\varsigma, v)
$$

for all $\varsigma, v \in \wp$, where $\sigma$ is a nonnegative real number with $\sigma<1$. Choose $\varsigma_{n}=\mho^{n} \varsigma_{\circ}$ for $\varsigma_{0} \in$ $\wp$, then $\partial$ has a unique fixed point, provided that the following assumptions are satisfied:

- $\sup _{l \geq 1} \lim _{j \rightarrow \infty} \frac{I\left(\varsigma_{j+1}, \varsigma_{j+2}\right)}{J\left(s_{j}, \varsigma_{j+1}\right)} v\left(\varsigma_{j+1}, \varsigma_{l}\right)<\frac{1}{\sigma}$;

- $\lim _{n \rightarrow \infty} I\left(\varsigma, \varsigma_{n}\right)$ and $\lim _{n \rightarrow \infty} v\left(\varsigma, \varsigma_{n}\right)$ exist and are finite for all $\varsigma \in \wp$.

Another direction to obtain a cfp of $\circlearrowright$ and $\mho$ is by considering a strong contractive condition in the following theorem.

Theorem 2.8 Let $\left(\wp, \eta_{\beth}^{\nu}\right)$ be a complete $\eta_{\beth}^{\nu}$-metric space, and let the mappings $\circlearrowright, \mho: \wp \rightarrow \wp$ satisfy

$$
\eta_{\beth}^{v}(\partial \varsigma, \mho v) \leq(\Pi(\varsigma)-\Pi(\partial \varsigma)) \beth(\varsigma, v),
$$

where

$$
\beth(\varsigma, v)=\max \left\{\begin{array}{l}
\eta_{\beth}^{v}(\varsigma, v), \eta_{\beth}^{v}(\varsigma, \partial \varsigma), \eta_{\beth}^{v}(v, \mho v), \\
\frac{\eta_{\beth}^{\nu}(\varsigma, \partial \varsigma)+\eta \beth(v, \mho v)}{2}, \frac{\sqrt{\eta_{\beth}^{\nu}(v, \partial \varsigma) \cdot \eta_{\beth}^{\nu}(\varsigma, \mho v)}}{2}
\end{array}\right\},
$$

and $\Pi: \wp \rightarrow \mathbb{R}$ is bounded from below $(\inf \{\Pi(\wp)\}>-\infty)$. Assume that $\varsigma_{n}=\mho^{n} \varsigma_{\circ}$ for $\varsigma_{\circ} \in$ $\wp$, and

$$
\sup _{l \geq 1} \lim _{j \rightarrow \infty} \frac{I\left(\varsigma_{j+1}, \varsigma_{j+2}\right)}{I\left(\varsigma_{j}, \varsigma_{j+1}\right)} \nu\left(\varsigma_{j+1}, \varsigma_{l}\right)<\frac{1}{\aleph}, \quad 0<\aleph<1 .
$$

In addition, suppose that $\lim _{n \rightarrow \infty} I\left(\varsigma, \varsigma_{n}\right)$ and $\lim _{n \rightarrow \infty} v\left(\varsigma, \varsigma_{n}\right)$ exist and are finite for all $\varsigma \in \wp$. Then $\partial$ and $\mho$ have a unique cfp.

Proof As at the beginning of the proof of Theorem 2.5, we consider the same sequences defined by (2.3). Consider

$$
\eta_{\beth}^{v}\left(\varsigma_{2 n+1}, \varsigma_{2 n+2}\right)=\eta_{\beth}^{v}\left(\circlearrowright \varsigma_{2 n}, \mho \varsigma_{2 n+1}\right) \leq\left(\Pi\left(\varsigma_{2 n}\right)-\Pi\left(\circlearrowright \varsigma_{2 n}\right)\right) \beth\left(\varsigma_{2 n}, \varsigma_{2 n+1}\right),
$$

where

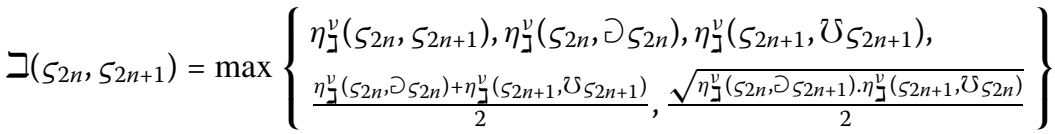

$$
\begin{aligned}
& =\max \left\{\begin{array}{l}
\eta_{\beth}^{\nu}\left(\varsigma_{2 n}, \varsigma_{2 n+1}\right), \eta_{\beth}^{\nu}\left(\varsigma_{2 n}, \varsigma_{2 n+1}\right), \eta_{\beth}^{\nu}\left(\varsigma_{2 n+1}, \varsigma_{2 n+2}\right), \\
\frac{\eta_{\beth}^{\nu}\left(\varsigma_{2 n}, \varsigma_{2 n+1}\right)+\eta_{\beth}^{\nu}\left(\varsigma_{2 n+1}, \varsigma_{2 n+2}\right)}{2}, \frac{\sqrt{\eta_{\beth}^{\nu}\left(\varsigma_{2 n+1}, \varsigma_{2 n+1}\right) \cdot \eta_{\beth}^{\nu}\left(\varsigma_{2 n}, \varsigma_{2 n+2}\right)}}{2}
\end{array}\right\} \\
& =\max \left\{\eta_{\beth}^{\nu}\left(\varsigma_{2 n}, \varsigma_{2 n+1}\right), \eta_{\beth}^{\nu}\left(\varsigma_{2 n+1}, \varsigma_{2 n+2}\right)\right\} \text {. }
\end{aligned}
$$


Now, if $\eta_{\beth}^{v}\left(\varsigma_{2 n+1}, \varsigma_{2 n+2}\right) \geq \eta_{\beth}^{v}\left(\varsigma_{2 n+1}, \varsigma_{2 n+2}\right)$, then by $(2.10)$ we get

$$
\begin{aligned}
\eta_{\beth}^{\nu}\left(\varsigma_{2 n+1}, \varsigma_{2 n+2}\right) & =\eta_{\beth}^{\nu}\left(\varsigma_{2 n}, \mho \varsigma_{2 n+1}\right) \\
& \leq\left(\Pi\left(\varsigma_{2 n}\right)-\Pi\left(\partial \varsigma_{2 n}\right)\right) \eta_{\beth}^{\nu}\left(\varsigma_{2 n+1}, \varsigma_{2 n+2}\right) \\
& =\left(\Pi\left(\varsigma_{2 n}\right)-\Pi\left(\varsigma_{2 n+1}\right)\right) \eta_{\beth}^{\nu}\left(\varsigma_{2 n+1}, \varsigma_{2 n+2}\right),
\end{aligned}
$$

a contradiction. So, $\beth\left(\varsigma_{2 n}, \varsigma_{2 n+1}\right)=\eta_{\beth}^{\nu}\left(\varsigma_{2 n}, \varsigma_{2 n+1}\right)$. Again, by (2.10), one can write

$$
\eta_{\beth}^{\nu}\left(\varsigma_{2 n+1}, \varsigma_{2 n+2}\right)=\eta_{\beth}^{v}\left(\partial \varsigma_{2 n}, \mho \varsigma_{2 n+1}\right) \leq\left(\Pi\left(\varsigma_{2 n}\right)-\Pi\left(\varsigma_{2 n+1}\right)\right) \eta_{\beth}^{v}\left(\varsigma_{2 n}, \varsigma_{2 n+1}\right) .
$$

Similarly,

$$
\eta_{\beth}^{\nu}\left(\varsigma_{2 n+2}, \varsigma_{2 n+3}\right)=\eta_{\beth}^{\nu}\left(\partial \varsigma_{2 n+1}, \mho \varsigma_{2 n+2}\right) \leq\left(\Pi\left(\varsigma_{2 n+1}\right)-\Pi\left(\varsigma_{2 n+2}\right)\right) \beth\left(\varsigma_{2 n+1}, \varsigma_{2 n+2}\right),
$$

where

$$
\begin{aligned}
& \beth\left(\varsigma_{2 n+1}, \varsigma_{2 n+2}\right)=\max \left\{\begin{array}{l}
\eta_{\beth}^{\nu}\left(\varsigma_{2 n+1}, \varsigma_{2 n+2}\right), \eta_{\beth}^{\nu}\left(\varsigma_{2 n+1}, \partial \varsigma_{2 n+1}\right), \eta_{\beth}^{\nu}\left(\varsigma_{2 n+2}, \mho \varsigma_{\varsigma_{2 n+2}}\right), \\
\frac{\eta_{\beth}^{\nu}\left(\varsigma_{2 n+1}, \partial \varsigma_{2 n+1}\right)+\eta_{\beth}^{\nu}\left(\varsigma_{2 n+2}, \mho \varsigma_{2 n+2}\right)}{2}, \frac{\sqrt{\eta_{\beth}^{\nu}\left(\varsigma_{2 n+1}, \partial \varsigma_{2 n+2}\right) \cdot \eta_{\beth}^{\nu}\left(\varsigma_{2 n+2}, \mho_{\left.\varsigma_{2 n+1}\right)}\right.}}{2}
\end{array}\right\}
\end{aligned}
$$

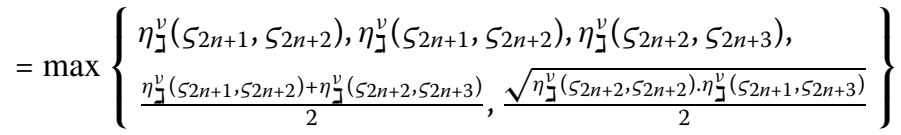

$$
\begin{aligned}
& =\max \left\{\eta_{\beth}^{\nu}\left(\varsigma_{2 n+1}, \varsigma_{2 n+2}\right), \eta_{\beth}^{\nu}\left(\varsigma_{2 n+2}, \varsigma_{2 n+3}\right)\right\} \text {. }
\end{aligned}
$$

From (2.11), we have

$$
\eta_{\beth}^{v}\left(\varsigma_{2 n+2}, \varsigma_{2 n+3}\right)=\eta_{\beth}^{v}\left(\partial \varsigma_{2 n+1}, \mho_{\varsigma_{2 n+2}}\right) \leq\left(\Pi\left(\varsigma_{2 n+1}\right)-\Pi\left(\varsigma_{2 n+2}\right)\right) \eta_{\beth}^{v}\left(\varsigma_{2 n+2}, \varsigma_{2 n+3}\right),
$$

a contradiction. Hence, $\beth\left(\varsigma_{2 n+1}, \varsigma_{2 n+2}\right)=\eta_{\beth}^{v}\left(\varsigma_{2 n+1}, \varsigma_{2 n+2}\right)$, then by $(2.11)$ we get

$$
\eta_{\beth}^{\nu}\left(\varsigma_{2 n+2}, \varsigma_{2 n+3}\right)=\eta_{\beth}^{\nu}\left(\partial \varsigma_{2 n+1}, \mho_{\varsigma_{2 n+2}}\right) \leq\left(\Pi\left(\varsigma_{2 n+1}\right)-\Pi\left(\varsigma_{2 n+2}\right)\right) \eta_{\beth}^{\nu}\left(\varsigma_{2 n+1}, \varsigma_{2 n+2}\right) .
$$

Continuing this approach, for each $n \in \mathbb{N}$, we conclude that

$$
\eta_{\beth}^{\nu}\left(\varsigma_{n}, \varsigma_{n+1}\right) \leq\left(\Pi\left(\varsigma_{n-1}\right)-\Pi\left(\varsigma_{n}\right)\right) \eta_{\beth}^{\nu}\left(\varsigma_{n-1}, \varsigma_{n}\right) .
$$

That is,

$$
\frac{\eta_{\beth}^{v}\left(\varsigma_{n}, \varsigma_{n+1}\right)}{\eta_{\beth}^{v}\left(\varsigma_{n-1}, \varsigma_{n}\right)} \leq\left(\Pi\left(\varsigma_{n-1}\right)-\Pi\left(\varsigma_{n}\right)\right) .
$$

Thus, the sequence $\left\{\zeta_{n}\right\}$ is necessarily decreasing and positive. So, it converges to an element $\mathfrak{s} \geq 0$. Mathematically, we get, for each $n \in \mathbb{N}$,

$$
\begin{aligned}
\sum_{\pi=1}^{n} \frac{\eta_{\Xi}^{\nu}\left(\varsigma_{\pi}, \varsigma_{\pi+1}\right)}{\eta_{\beth}^{v}\left(\varsigma_{\pi-1}, \varsigma_{\pi}\right)} & \leq \sum_{\pi=1}^{n}\left(\Pi\left(\varsigma_{\pi-1}\right)-\Pi\left(\varsigma_{\pi}\right)\right) \\
& =\left(\Pi\left(\varsigma_{0}\right)-\Pi\left(\varsigma_{1}\right)\right)+\left(\Pi\left(\varsigma_{1}\right)-\Pi\left(\varsigma_{2}\right)\right)+\cdots+\left(\Pi\left(\varsigma_{n-1}\right)-\Pi\left(\varsigma_{n}\right)\right) \\
& =\Pi\left(\varsigma_{0}\right)-\Pi\left(\varsigma_{n}\right) \rightarrow \Pi\left(\varsigma_{0}\right)-\Im \text { as } n \rightarrow \infty,
\end{aligned}
$$




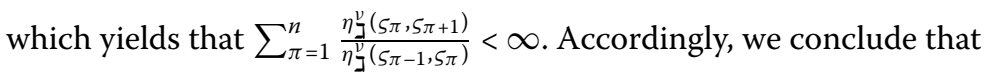

$$
\lim _{n \rightarrow \infty} \frac{\eta_{\beth}^{\nu}\left(\varsigma_{\pi}, \varsigma_{\pi+1}\right)}{\eta_{\beth}^{\nu}\left(\varsigma_{\pi-1}, \varsigma_{\pi}\right)}=0
$$

It follows by $(2.12)$ that there is $0<\aleph<1$ so that

$$
\frac{\eta_{\beth}^{\nu}\left(\varsigma_{\pi}, \varsigma_{\pi+1}\right)}{\eta_{\beth}^{\nu}\left(\varsigma_{\pi-1}, \varsigma_{\pi}\right)} \leq \aleph \quad \text { for all } \pi \geq \pi_{0}
$$

Equivalently,

$$
\eta_{\beth}^{v}\left(\varsigma_{\pi}, \varsigma_{\pi+1}\right) \leq \aleph \eta_{\beth}^{v}\left(\varsigma_{\pi-1}, \varsigma_{\pi}\right) \text { for all } \pi \geq \pi_{0} .
$$

By continuing with the same scenario used in proving Theorem 2.5, we conclude that the sequence $\left\{\zeta_{n}\right\}$ converges to some $\vartheta \in \wp$, i.e.,

$$
\lim _{n \rightarrow \infty} \eta_{\beth}^{\nu}\left(\varsigma_{n}, \vartheta\right)=0
$$

It is clear that

$$
\begin{aligned}
\eta_{\beth}^{\nu}\left(\varsigma_{2 n+1}, \varsigma_{2 n+2}\right) & \leq \beth\left(\varsigma_{2 n+1}, \vartheta\right) \eta_{\beth}^{\nu}\left(\varsigma_{2 n+1}, \vartheta\right)+\nu\left(\vartheta, \varsigma_{2 n+2}\right) \eta_{\beth}^{\nu}\left(\vartheta, \varsigma_{2 n+2}\right) \\
& \rightarrow 0 \quad \text { as } n \rightarrow \infty .
\end{aligned}
$$

Now, we show that $\vartheta=\partial \vartheta$. Suppose to the contrary, i.e., $\eta_{\beth}^{v}(\partial \vartheta, \vartheta)>0$. Consider

$$
\begin{aligned}
\eta_{\beth}^{\nu}(\vartheta, \partial \vartheta) & \leq \beth\left(\vartheta, \varsigma_{2 n+2}\right) \eta_{\beth}^{\nu}\left(\vartheta, \varsigma_{2 n+2}\right)+v\left(\varsigma_{2 n+2}, \partial \vartheta\right) \eta_{\beth}^{\nu}\left(\varsigma_{2 n+2}, \partial \vartheta\right) \\
& =\beth\left(\vartheta, \varsigma_{2 n+2}\right) \eta_{\beth}^{v}\left(\vartheta, \varsigma_{2 n+2}\right)+v\left(\varsigma_{2 n+2}, \partial \vartheta\right) \eta_{\beth}^{v}\left(\partial \vartheta, \mho \varsigma_{2 n+1}\right) \\
& \leq \beth\left(\vartheta, \varsigma_{2 n+2}\right) \eta_{\beth}^{v}\left(\vartheta, \varsigma_{2 n+2}\right)+v\left(\varsigma_{2 n+2}, \partial \vartheta\right)(\Pi(\vartheta)-\Pi(\partial \vartheta)) \beth\left(\vartheta, \varsigma_{2 n+1}\right),
\end{aligned}
$$

where

$$
\begin{aligned}
& \beth\left(\vartheta, \varsigma_{2 n+1}\right)=\max \left\{\begin{array}{l}
\eta_{\beth}^{v}\left(\vartheta, \varsigma_{2 n+1}\right), \eta_{\beth}^{\nu}(\vartheta, \partial \vartheta), \eta_{\beth}^{\nu}\left(\varsigma_{2 n+1}, \mho \varsigma_{2 n+1}\right), \\
\frac{\eta_{\beth}^{\nu}(\vartheta, \partial \vartheta)+\eta_{\beth}^{\nu}\left(\varsigma_{2 n+1}, \mho \mho_{52 n+1}\right)}{2}, \frac{\sqrt{\eta_{\beth}^{\nu}\left(\varsigma_{2 n+1}, \partial \vartheta\right) \cdot \eta_{\beth}^{\nu}\left(\vartheta, \mho \mho_{52 n+1}\right)}}{2}
\end{array}\right\} \\
& =\max \left\{\begin{array}{l}
\eta_{\beth}^{\nu}\left(\vartheta, \varsigma_{2 n+1}\right), \eta_{\beth}^{\nu}(\vartheta, \partial \vartheta), \eta_{\beth}^{\nu}\left(\varsigma_{2 n+1}, \varsigma_{2 n+2}\right), \\
\frac{\eta_{\beth}^{v}(\vartheta, \partial \vartheta)+\eta_{J}^{\nu}\left(\varsigma_{2 n+1}, \varsigma_{2 n+2}\right)}{2}, \frac{\sqrt{\eta_{\beth}^{\nu}\left(\varsigma_{2 n+1}, \partial \vartheta\right) \cdot \eta_{\beth}^{\nu}\left(\vartheta, \varsigma_{2 n+2}\right)}}{2}
\end{array}\right\} .
\end{aligned}
$$

Letting $n \rightarrow \infty$ in (2.16) and applying (2.13) and (2.14), we have

$$
\lim _{n \rightarrow \infty} \beth\left(\vartheta, \varsigma_{2 n+1}\right)=\eta_{\beth}^{v}(\vartheta, \partial \vartheta)
$$

Taking $n \rightarrow \infty$ in (2.15) and by (2.17), we get

$$
\eta_{\beth}^{\nu}(\vartheta, \partial \vartheta) \leq v(\vartheta, \partial \vartheta)(\Pi(\vartheta)-\Pi(\partial \vartheta)) \eta_{\beth}^{\nu}(\vartheta, \partial \vartheta),
$$


a contradiction. Hence, $\eta_{\beth}^{v}(\vartheta, \partial \vartheta)=0$, i.e., $\vartheta=\partial \vartheta$. Similarly, we can show that $\vartheta=\mho \vartheta$. To prove the uniqueness of the $\operatorname{cfp}$ of $\partial$ and $\mho$, let $\omega \neq \vartheta$ be another $\operatorname{cfp}$ of $\partial$ and $\mho$, i.e., $\eta_{\beth}^{\nu}(\vartheta, \omega)>0$. Consider

$$
\begin{aligned}
& \eta_{\beth}^{v}(\vartheta, \omega)=\eta_{\beth}^{v}(\partial \vartheta, \mho \omega) \\
& \leq(\Pi(\vartheta)-\Pi(\partial \vartheta)) \beth(\vartheta, \omega) \\
& =(\Pi(\vartheta)-\Pi(\partial \vartheta)) \max \left\{\begin{array}{l}
\eta_{\beth}^{\nu}(\vartheta, \omega), \eta_{\beth}^{\nu}(\vartheta, \partial \vartheta), \eta_{\beth}^{\nu}(\omega, \mho \omega), \\
\frac{\eta_{\beth}^{\nu}(\vartheta, \partial \vartheta)+\eta_{\beth}^{\nu}(\omega, \mho \omega)}{2}, \frac{\sqrt{\eta_{\beth}^{\nu}(\omega, \partial \vartheta) \cdot \eta_{\beth}^{\nu}(\vartheta, \mho \mho \omega)}}{2}
\end{array}\right\} \\
& =(\Pi(\vartheta)-\Pi(\partial \vartheta)) \max \left\{\begin{array}{l}
\eta_{\beth}^{\nu}(\vartheta, \omega), \eta_{\beth}^{\nu}(\vartheta, \vartheta), \eta_{\beth}^{\nu}(\omega, \omega), \\
\frac{\eta_{\beth}^{\nu}(\vartheta, \vartheta)+\eta_{\beth}^{\nu}(\omega, \omega)}{2}, \frac{\sqrt{\eta_{\beth}^{\nu}(\omega, \vartheta) \cdot \eta_{\beth}^{\nu}(\vartheta, \omega)}}{2}
\end{array}\right\} \\
& =(\Pi(\vartheta)-\Pi(\partial \vartheta)) \max \left\{\eta_{\beth}^{v}(\vartheta, \omega), 0,0,0, \frac{\eta_{\beth}^{\nu}(\vartheta, \omega)}{2}\right\} \\
& =(\Pi(\vartheta)-\Pi(\supset \vartheta)) \eta_{\beth}^{v}(\vartheta, \omega) \text {. }
\end{aligned}
$$

There exists a contradiction in the relation $\eta_{\beth}^{\nu}(\vartheta, \omega) \leq(\Pi(\vartheta)-\Pi(\circlearrowright \vartheta)) \eta_{\beth}^{\nu}(\vartheta, \omega)$, thus $\eta_{\beth}^{v}(\vartheta, \omega)=0$, i.e., $\vartheta=\omega$. Therefore, $\vartheta$ is the unique cfp of the mappings $\partial$ and $\mho$.

Example 2.9 Assume that all requirements of Example $2.2 \mathrm{hold}$, then $\left(\wp, \eta_{\mathrm{J}}^{\nu}\right)$ is a complete $\eta_{\beth}^{\nu}$-metric space. Let the mappings $\partial, \mho: \wp \rightarrow \wp$ be defined by $\partial 1=\partial 3=1, \partial 2=3$ and $\mho 1=\mho 2=1, \mho 3=2$, respectively. Now, we shall verify the contractive condition (2.1) of Theorem 2.5. For $a=1, b=2, \sigma=\frac{1}{2}, \rho=\frac{1}{4}$, and $\mu=\frac{1}{5}$ (it is obvious that $\sigma+\rho+\mu=\frac{19}{20}<1$ ), for simplicity, we put

$$
\Omega(\varsigma, v)=\sigma \eta_{\beth}^{v}(\varsigma, v)+\rho \frac{\eta_{\beth}^{v}(v, \mho v)\left[1+\eta_{\beth}^{v}(v, \mho v)\right]}{1+\eta_{\beth}^{v}(\partial \varsigma, \mho v)}+\mu \frac{\eta_{\beth}^{v}(v, \mho v)\left[1+\eta_{\beth}^{v}(\varsigma, \partial \varsigma)\right]}{1+\eta_{\beth}^{v}(\varsigma, v)} .
$$

We consider the following cases:

- If $\varsigma=v=1$ or $(\varsigma=1, v=2)$, or $(\varsigma=3, v=2)$, or $(\varsigma=3, v=1)$. This case is achieved directly because of

$$
\eta_{\beth}^{\nu}(\partial 1, \mho 1)=\eta_{\beth}^{\nu}(\partial 1, \mho 2)=\eta_{\beth}^{\nu}(\partial 3, \mho 2)=\eta_{\beth}^{\nu}(\partial 3, \mho 1)=\eta_{\beth}^{\nu}(1,1)=0 .
$$

- If $\varsigma=v=2$, then

$$
a^{b} \eta_{\beth}^{\nu}\left(\supset_{\zeta}, \mho v\right)=\eta_{\beth}^{\nu}(\supset 2, \mho 2)=\eta_{\beth}^{\nu}(3,1)=1
$$

and

$$
\Omega(\varsigma, v)=\Omega(2,2)=0 \sigma+3 \rho+10 \mu=3\left(\frac{1}{4}\right)+10\left(\frac{1}{5}\right)=2.75 .
$$

It is easily seen that $a^{b} \eta_{\beth}^{v}(\partial \varsigma, \mho v) \leq \Omega(\varsigma, v)$.

- If $\varsigma=v=3$, then

$$
a^{b} \eta_{\beth}^{\nu}\left(\supset_{\zeta}, \mho v\right)=\eta_{\beth}^{\nu}(\circlearrowright 3, \mho 3)=\eta_{\beth}^{\nu}(1,2)=2
$$


and

$$
\Omega(\varsigma, v)=\Omega(3,3)=0 \sigma+5.25 \rho+7.2 \mu=5.25\left(\frac{1}{4}\right)+7.2\left(\frac{1}{5}\right)=2.7525
$$

So, we have $a^{b} \eta_{\beth}^{\nu}\left(\zeta_{\zeta}, \mho v\right) \leq \Omega(\varsigma, v)$.

- If $\varsigma=1, v=3$, then

$$
\left.a^{b} \eta_{\beth}^{v}()_{\zeta}, \mho v\right)=\eta_{\beth}^{v}(\circlearrowright 1, \mho 3)=\eta_{\beth}^{v}(1,2)=2
$$

and

$$
\Omega(\varsigma, v)=\Omega(1,3)=\sigma+\frac{20}{3} \rho+2 \mu=\frac{1}{2}+\frac{20}{3}\left(\frac{1}{4}\right)+2\left(\frac{1}{5}\right)=2.566666667
$$

hence, we observe that $a^{b} \eta_{\beth}^{v}(\partial \varsigma, \mho v) \leq \Omega(\varsigma, v)$.

- If $\varsigma=2, v=1$, then

$$
\left.a^{b} \eta_{\beth}^{v}()_{\zeta}, \mho v\right)=\eta_{\beth}^{\nu}(\supset 2, \mho 1)=\eta_{\beth}^{v}(3,1)=1
$$

and

$$
\Omega(\varsigma, v)=\Omega(2,1)=2 \sigma+0 \rho+0 \mu=2\left(\frac{1}{2}\right)=1 .
$$

Thus, $a^{b} \eta_{\jmath}^{\nu}(\partial \varsigma, \mho v) \leq \Omega(\varsigma, v)$.

- If $\varsigma=2$ and $v=3$, then

$$
a^{b} \eta_{\beth}^{\nu}(\partial \varsigma, \mho v)=\eta_{\beth}^{\nu}(\partial 2, \mho 3)=\eta_{\beth}^{\nu}(3,2)=3.6
$$

and

$$
\Omega(\varsigma, v)=\Omega(2,3)=4(\sigma+\rho+\mu)=3.8 .
$$

It is clear that $a^{b} \eta_{\beth}^{v}\left(\supset_{\varsigma}, \mho v\right) \leq \Omega(\varsigma, v)$.

From the above cases, the hypotheses managed by Theorem 2.5 are fulfilled, and 1 is the unique cfp of $\partial$ and $\mho$.

Example 2.10 Suppose that the data of Example 2.2 are verified, then ( $\left.\wp, \eta_{\beth}^{\nu}\right)$ is a complete $\eta_{\mathrm{J}}^{\nu}$-metric space. Suppose that the mappings $\partial, \mho: \wp \rightarrow \wp$ are defined by $\partial 1=\partial 2=2$, $\partial 3=\mho 1=1$ and $\mho 2=\mho 3=2$. Define $\Pi: \wp \rightarrow[0, \infty)$ as $\Pi(1)=4, \Pi(2)=3$, and $\Pi(3)=5$. Thus, for all $\varsigma, v \in \wp$ such that $\varsigma \neq 2$, we discuss the following cases:

$\Delta$ If $(\varsigma=1, v=3)$ or $(\varsigma=1, v=2)$, or $(\varsigma=3, v=1)$, we have $\eta_{\jmath}^{\nu}(\circlearrowright 1, \mho 3)=\eta_{\jmath}^{\nu}(\circlearrowright 1, \mho 2)=$ $\eta_{\beth}^{\nu}(2,2)=0$ and $\eta_{\beth}^{\nu}(\partial 3, \mho 1)=\eta_{\beth}^{\nu}(1,1)=0$, respectively. So this case is trivial.

\ If $\varsigma=v=1$, we have $\eta_{\beth}^{v}(\partial 1, \mho 1)=\eta_{\beth}^{v}(2,1)=2$. 
Consider

$$
\begin{aligned}
& (\Pi(1)-\Pi(\circlearrowright 1)) \beth(1,1)=(4-3) \beth(1,1) \\
& =\max \left\{\begin{array}{l}
\eta_{\beth}^{\nu}(1,1), \eta_{\beth}^{\nu}(1, \circlearrowright 1), \eta_{\beth}^{\nu}(1, \mho 1), \\
\frac{\eta_{\beth}^{\nu}(1, \partial 1)+\eta_{\beth}^{\nu}(1, \mho 1)}{2}, \frac{\sqrt{\eta_{\beth}^{\nu}(1, \partial 1) \cdot \eta_{\beth}^{\nu}(1, \mho 1)}}{2}
\end{array}\right\} \\
& =\max \left\{\begin{array}{l}
\eta_{\beth}^{\nu}(1,1), \eta_{\beth}^{\nu}(1,2), \eta_{\beth}^{\nu}(1,1), \\
\frac{\eta_{\beth}^{\nu}(1,2)+\eta_{\beth}^{\nu}(1,1)}{2}, \frac{\sqrt{\eta_{\beth}^{\nu}(1,2) \cdot \eta_{\beth}^{\nu}(1,1)}}{2}
\end{array}\right\} \\
& =\max \{0,2,0,1,0\}=2 \text {. }
\end{aligned}
$$

This leads to $\eta_{\beth}^{v}(\circlearrowright 1, \mho 1) \leq(\Pi(1)-\Pi(\circlearrowright 1)) \beth(1,3)$.

\ If $\varsigma=v=3$, we have $\eta_{\beth}^{v}(\supset 3, \mho 3)=\eta_{\beth}^{v}(1,2)=2$.

Consider

$$
\begin{aligned}
& (\Pi(3)-\Pi(\circlearrowright 3)) \beth(3,3)=(5-4) \beth(3,3) \\
& =\max \left\{\begin{array}{l}
\eta_{\beth}^{\nu}(3,3), \eta_{\beth}^{\nu}(3, \circlearrowright 3), \eta_{\beth}^{\nu}(3, \mho 3), \\
\frac{\eta_{\beth}^{\nu}(3, \circlearrowright 3)+\eta_{\beth}^{\nu}(3, \mho 3)}{2}, \frac{\sqrt{\eta_{\beth}^{\nu}(3, \supset 3) \cdot \eta_{\beth}^{\nu}(3, \mho 3)}}{2}
\end{array}\right\} \\
& =\max \left\{\begin{array}{l}
\eta_{\beth}^{\nu}(3,3), \eta_{\beth}^{\nu}(3,1), \eta_{\beth}^{\nu}(3,2), \\
\frac{\eta_{\beth}^{\nu}(3,1)+\eta_{\beth}^{\nu}(3,2)}{2}, \frac{\sqrt{\eta_{\beth}^{\nu}(3,1) \cdot \eta_{\beth}^{\nu}(3,2)}}{2}
\end{array}\right\} \\
& =\max \left\{0,1,3.6,2.3, \frac{3 \sqrt{10}}{10}\right\}=3.6 \text {. }
\end{aligned}
$$

It yields that $\eta_{\beth}^{\nu}(\supset 3, \mho 3) \leq(\Pi(3)-\Pi(\circlearrowright 3)) \beth(3,3)$.

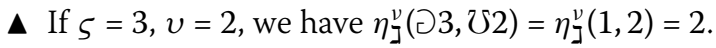

Consider

$$
\begin{aligned}
& (\Pi(3)-\Pi(\circlearrowright 3)) \beth(3,2)=(5-4) \beth(3,2) \\
& =\max \left\{\begin{array}{l}
\eta_{\beth}^{\nu}(3,2), \eta_{\beth}^{\nu}(3, \circlearrowright 3), \eta_{\beth}^{\nu}(2, \mho 2), \\
\frac{\eta_{\beth}^{\nu}(3, \circlearrowright 3)+\eta_{\beth}^{\nu}(2, \mho 2)}{2}, \frac{\sqrt{\eta_{\beth}^{\nu}(2, \supset 3) \cdot \eta_{\beth}^{\nu}(3, \mho 2)}}{2}
\end{array}\right\} \\
& =\max \left\{\begin{array}{l}
\eta_{\beth}^{\nu}(3,2), \eta_{\beth}^{\nu}(3,1), \eta_{\beth}^{\nu}(2,2), \\
\frac{\eta_{\beth}^{\nu}(3,1)+\eta_{\beth}^{\nu}(2,2)}{2}, \frac{\sqrt{\eta_{\beth}^{\nu}(2,1) \cdot \eta_{\beth}^{\nu}(3,2)}}{2}
\end{array}\right\} \\
& =\max \left\{3.6,1,0,0.5, \frac{3 \sqrt{5}}{5}\right\}=3.6 \text {. }
\end{aligned}
$$

This implies that $\eta_{\beth}^{\nu}(\supset 3, \mho 3) \leq(\Pi(3)-\Pi(\supset 3)) \beth(3,2)$.

According to the above cases, we observe that all assumptions of Theorem 2.8 are fulfilled and 2 is the unique cfp of $\partial$ and $\mho$.

\section{Solving the 2D Volterra integral equations}

There are many advantages to studying equations of the form (3.1). The authors [37] showed that problem (3.1) arises from the transformation of certain Volterra integral 
equations of the first kind, with applications, for example, in analysis of Cauchy problems for certain partial differential equations (e.g., the telegraph equation) and in radiation transfer problems. Moreover, the Darboux problem can also be reduced to equation (3.1), as shown in [38].

In this section, we highlight the role of this technique in finding a solution of the twodimensional (2D) Volterra integral equations, which takes the form

$$
\begin{aligned}
\Lambda(\lambda, \hbar)= & \xi(\lambda, \hbar)+\int_{0}^{\lambda} \int_{0}^{\hbar} \Xi_{1}(\varsigma, v, \Lambda(\varsigma, v)) d \varsigma d v \\
& +\beth \int_{0}^{\lambda} \Xi_{2}(\hbar, v, \Lambda(\lambda, v)) d v+v \int_{0}^{\hbar} \Xi_{3}(\lambda, \varsigma, \Lambda(\hbar, \varsigma)) d \varsigma
\end{aligned}
$$

for all $\lambda, \hbar, \varsigma, v \in[0,1]$, where $\Lambda \in \wp=C([0,1] \times[0,1])$ and $\xi:[0,1] \times[0,1] \rightarrow \mathbb{R}^{2} ; \Xi_{i}(i=$ $1,2,3):[0,1] \times[0,1] \times \mathbb{R}^{2} \rightarrow \mathbb{R}^{2}$. Define the distance $\eta_{]}^{v}: \wp \times \wp \rightarrow[0, \infty)$ on the set of all continuous functions $\wp=C\left([0,1]^{2}, \mathbb{R}^{+}\right)$from $[0,1]^{2}$ onto $\mathbb{R}^{+}$as follows:

$$
\eta_{\beth}^{\nu}(\Lambda(\lambda, \hbar), \varpi(\lambda, \hbar))=|\kappa(\lambda, \hbar)-\varpi(\lambda, \hbar)|^{2}
$$

for all $\kappa, \varpi \in \wp$. Let the functions $\beth, v: \wp \times \wp \rightarrow[1, \infty)$ be defined by

$$
\begin{aligned}
& I(\Lambda(\lambda, \hbar), \varpi(\lambda, \hbar))=\frac{|\Lambda(\lambda, \hbar)|+|\varpi(\lambda, \hbar)|}{2}+2, \\
& v(\Lambda(\lambda, \hbar), \varpi(\lambda, \hbar))=\frac{|\Lambda(\lambda, \hbar)|+|\varpi(\lambda, \hbar)|}{1+|\Lambda(\lambda, \hbar)|+|\varpi(\lambda, \hbar)|}+2 .
\end{aligned}
$$

Then $\left(\wp, \eta_{\beth}^{\nu}\right)$ is clearly an $\eta_{\beth}^{\nu}$-metric space.

We shall consider problem (3.1) via the following assumptions:

(i) $\Xi_{i}(i=1,2,3):[0,1] \times[0,1] \times \mathbb{R}^{2} \rightarrow \mathbb{R}^{2}$ are continuous functions satisfying

$$
\begin{aligned}
& \mid \Xi_{1}\left(\varsigma, v, \hbar_{1}(\varsigma, v)-\Xi_{1}\left(\varsigma, v, \hbar_{2}(\varsigma, v) \mid \leq ¥ 1 \sqrt{\left|\hbar_{1}(\varsigma, v)-\hbar_{2}(\varsigma, v)\right|},\right.\right. \\
& \mid \Xi_{2}\left(\varsigma, v, \hbar_{1}(\varsigma, v)-\Xi_{2}\left(\varsigma, v, \hbar_{2}(\varsigma, v) \mid \leq ¥{ }_{2} \sqrt{\left|\hbar_{1}(\varsigma, v)-\hbar_{2}(\varsigma, v)\right|},\right.\right. \\
& \mid \Xi_{3}\left(\varsigma, v, \hbar_{1}(\varsigma, v)-\Xi_{3}\left(\varsigma, v, \hbar_{2}(\varsigma, v) \mid \leq ¥ 3 \sqrt{\left|\hbar_{1}(\varsigma, v)-\hbar_{2}(\varsigma, v)\right|},\right.\right.
\end{aligned}
$$

for constants $¥_{1}, ¥_{2}, ¥_{3} \geq 0$ and $\hbar_{1}, \hbar_{2} \in \mathbb{R}^{2}$;

(ii) $¥_{1}+|\mathrm{I}| ¥_{2}+|v| ¥_{3} \leq \sigma$, where $0<\sigma<1$.

The important theorem of this part is showed below.

Theorem 3.1 Assume that the above conditions (i)-(ii) hold, then problem (3.1) has a unique solution.

Proof Define the integral operator $\partial: \wp \rightarrow \wp$ by

$$
\begin{aligned}
\partial \Lambda(\lambda, \hbar)= & \xi(\lambda, \hbar)+\int_{0}^{\lambda} \int_{0}^{\hbar} \Xi_{1}(\varsigma, v, \Lambda(\varsigma, v)) d \varsigma d v \\
& +\beth \int_{0}^{\lambda} \Xi_{2}(\hbar, v, \Lambda(\lambda, v)) d v+v \int_{0}^{\hbar} \Xi_{3}(\lambda, \varsigma, \Lambda(\hbar, \varsigma)) d \varsigma .
\end{aligned}
$$


It is obvious that from (3.2) a unique fixed point of the mapping $\partial$ is the unique solution of problem (3.1). By (3.2), we have

$$
\begin{aligned}
\eta_{\beth}^{v}( & \partial(\Lambda, \hbar)), \partial(\varpi(\lambda, \hbar))) \\
= & |\partial(\Lambda(\lambda, \hbar))-\partial(\varpi(\lambda, \hbar))|^{2} \\
= & \mid \xi(\lambda, \hbar)+\int_{0}^{\lambda} \int_{0}^{\hbar} \Xi_{1}(\varsigma, v, \Lambda(\varsigma, v)) d \varsigma d v+\Xi \int_{0}^{\lambda} \Xi_{2}(\hbar, v, \Lambda(\lambda, v)) d v \\
& +v \int_{0}^{\hbar} \Xi_{3}(\lambda \hbar, \varsigma, \Lambda(\hbar, \varsigma)) d \varsigma-\xi(\lambda, \hbar)-\int_{0}^{\lambda} \int_{0}^{\hbar} \Xi_{1}(\varsigma, v, \varpi(\varsigma, v)) d \varsigma d v \\
& -\Xi \int_{0}^{\lambda} \Xi_{2}(\hbar, v, \varpi(\lambda, v)) d v-\left.v \int_{0}^{\hbar} \Xi_{3}(\lambda, \varsigma, \varpi(\hbar, \varsigma)) d \varsigma\right|^{2} \\
= & \mid \int_{0}^{\lambda} \int_{0}^{\hbar}\left(\Xi_{1}(\varsigma, v, \Lambda(\varsigma, v))-\Xi_{1}(\varsigma, v, \varpi(\varsigma, v))\right) d \varsigma d v \\
& +\Xi \int_{0}^{\lambda}\left(\Xi_{2}(\hbar, v, \Lambda(\lambda, v))-\Xi_{2}(\hbar, v, \varpi(\lambda, v))\right) d v \\
& +\left.v \int_{0}^{\hbar}\left(\Xi_{3}(\lambda, \varsigma, \Lambda(\hbar, \varsigma))-\Xi_{3}(\lambda, \varsigma, \varpi(\hbar, \varsigma))\right) d \varsigma\right|^{2} \\
\leq & \left(\int_{0}^{\lambda} \int_{0}^{\hbar}\left|\Xi_{1}(\varsigma, v, \Lambda(\varsigma, v))-\Xi_{1}(\varsigma, v, \varpi(\varsigma, v))\right| d \varsigma d v\right. \\
& +|\Xi| \int_{0}^{\lambda}\left|\Xi_{2}(\hbar, v, \Lambda(\lambda, v))-\Xi_{2}(\hbar, v, \varpi(\lambda, v))\right| d v \\
& \left.+|v| \int_{0}^{\hbar}\left|\Xi_{3}(\lambda, \varsigma, \Lambda(\hbar, \varsigma))-\Xi_{3}(\lambda, \varsigma, \varpi(\hbar, \varsigma))\right| d \varsigma\right)^{2} .
\end{aligned}
$$

Applying the double conditions of our theorem, we get

$$
\begin{aligned}
& \eta_{\beth}^{\nu}(\partial(\Lambda(\lambda, \hbar)), \partial(\varpi(\lambda, \hbar))) \\
& \leq\left(\int_{0}^{\lambda} \int_{0}^{\hbar} ¥_{1} \sqrt{|\Lambda(\varsigma, v)-\varpi(\varsigma, v)|} d \varsigma d v+|\Xi| \int_{0}^{\lambda} ¥_{2} \sqrt{|\Lambda(\lambda, v)-\varpi(\lambda, v)|} d v\right. \\
& \left.+|v| \int_{0}^{\hbar} ¥ \sqrt{3|\Lambda(\hbar, \varsigma)-\varpi(\hbar, \varsigma)|} d \varsigma\right)^{2} \\
& \leq\left(¥_{1} \sqrt{|\Lambda(\varsigma, v)-\varpi(\varsigma, v)|}+|\exists| ¥_{2} \sqrt{|\Lambda(\lambda, v)-\varpi(\lambda, v)|}\right. \\
& \left.+|v| ¥_{3} \sqrt{|\Lambda(\hbar, \varsigma)-\varpi(\hbar, \varsigma)|}\right)^{2} \\
& \leq\left(¥_{1} \sqrt{|\Lambda(\lambda, \hbar)-\varpi(\lambda, \hbar)|}+|\exists| ¥_{2} \sqrt{|\Lambda(\lambda, \hbar)-\varpi(\lambda, \hbar)|}\right. \\
& \left.+|v| ¥_{3} \sqrt{|\Lambda(\lambda, \hbar)-\varpi(\lambda, \hbar)|}\right)^{2} \\
& =\left(\left(¥_{1}+|ב| ¥_{2}+|v| ¥_{3}\right) \sqrt{|\Lambda(\lambda, \hbar)-\varpi(\lambda, \hbar)|}\right)^{2} \\
& \leq \sigma^{2} \eta_{\beth}^{\nu}(\Lambda(\lambda, \hbar), \varpi(\lambda, \hbar)) \\
& \leq \sigma \eta_{\beth}^{\nu}(\Lambda(\lambda, \hbar), \varpi(\lambda, \hbar)) \text {. }
\end{aligned}
$$


Hence the requirements of Corollary 2.7 are fulfilled, therefore we observe that $\partial$ has a unique fixed point, and so problem (3.1) has a unique solution.

\section{A unique solution of Riemann-Liouville fractional integrals}

A bunch of scientists have tackled Riemann-Liouville integral equations and recently identified a new technique, so-called 'fixed point approach'. This novel approach has promised the existence of a solution of Riemann-Liouville integral equations. For more details, see $[39,40]$.

Along the same lines, here we study the existence and uniqueness of a solution of Riemann-Liouville (RL) fractional integral in the form of

$$
{ }_{\hbar}^{\mathrm{RL}} I_{\kappa}^{\tau} \zeta(\kappa)=\frac{1}{\Gamma(\tau)} \int_{\hbar}^{\kappa}(\kappa-\ell)^{\tau-1} \varsigma(\ell) d \ell ; \quad \Gamma(\tau)>0,
$$

where $\tau \in \mathbb{R}, \varsigma(\kappa) \in \wp=C([0,1], \mathbb{R})(C([0,1], \mathbb{R})$ is the set of all continuous functions from $[0,1]$ onto $\mathbb{R})$ and $\kappa, \ell \in[0,1]$ which is the fractional integral. Define the distance $\eta_{\beth}^{v}: \wp \times$ $\wp \rightarrow[0, \infty)$ by

$$
\eta_{\beth}^{\nu}(\varsigma, v)=|\varsigma(\kappa)-v(\kappa)|^{2}
$$

for all $\varsigma(\kappa), v(\kappa) \in \wp$ and $\kappa \in[0,1]$. Also define $], v: \wp \times \wp \rightarrow[1, \infty)$ by

$$
\begin{aligned}
& I(\varsigma(\kappa), v(\kappa))=\frac{|\zeta(\kappa)|+|v(\kappa)|}{2}+2, \\
& v(\varsigma(\kappa), v(\kappa))=\frac{|\zeta(\kappa)|+|v(\kappa)|}{1+|\zeta(\kappa)|+|v(\kappa)|}+2 .
\end{aligned}
$$

Then $\left(\wp, \eta_{\beth}^{\nu}\right)$ is an $\eta_{\beth}^{v}$-metric space.

Now, we shall show that integral (4.1) has a unique solution under the following condition:

$$
\frac{1}{\Gamma^{2}(\tau+1)} \frac{(\kappa-\ell)^{\tau-1}(\kappa-\hbar)^{2 \tau}}{\left|(\kappa-\ell)^{\tau-1}\right|}<\sigma, \quad \text { where } \sigma \in(0,1) \text { and } \kappa \neq \ell
$$

Define also the operator $\partial: \wp \rightarrow \wp$ by

$$
\partial \varsigma(\kappa)=\frac{1}{\Gamma(\tau)} \int_{\hbar}^{\kappa}(\kappa-\ell)^{\tau-1} \varsigma(\ell) d \ell
$$

Thus, the existence of a unique solution of problem (4.1) is equivalent to finding a unique fixed point of the integral operator (4.2).

Assume that

$$
\begin{aligned}
\eta_{\beth}^{v}(\partial \varsigma, \partial v) & =|\partial \varsigma(\kappa)-\partial v(\kappa)|^{2} \\
& =\left|\frac{1}{\Gamma(\tau)} \int_{\hbar}^{\kappa}(\kappa-\ell)^{\tau-1} \varsigma(\ell) d \ell-\frac{1}{\Gamma(\tau)} \int_{\hbar}^{\kappa}(\kappa-\ell)^{\tau-1} v(\ell) d \ell\right|^{2} \\
& \leq\left(\left|\frac{1}{\Gamma(\tau)} \int_{\hbar}^{\kappa}(\kappa-\ell)^{\tau-1} d \ell\right|\right)^{2}|\varsigma(\ell)-v(\ell)|^{2}
\end{aligned}
$$




$$
\begin{aligned}
& \leq \frac{1}{\Gamma^{2}(\tau)}\left(\int_{\hbar}^{\kappa}\left|(\kappa-\ell)^{\tau-1}\right| d \ell\right)^{2}|\varsigma(\ell)-v(\ell)|^{2} \\
& =\frac{1}{\Gamma^{2}(\tau)} \frac{(\kappa-\ell)^{\tau-1}}{\left|(\kappa-\ell)^{\tau-1}\right|}\left(\int_{\hbar}^{\kappa}(\kappa-\ell)^{\tau-1} d \ell\right)^{2}|\zeta(\ell)-v(\ell)|^{2} \\
& =\frac{-1}{\Gamma^{2}(\tau)} \frac{(\kappa-\ell)^{\tau-1}}{\left|(\kappa-\ell)^{\tau-1}\right|}\left(\left[\frac{(\kappa-\ell)^{\tau}}{\tau}\right]_{\hbar}^{\kappa}\right)^{2}|\zeta(\ell)-v(\ell)|^{2} \\
& =\frac{1}{\Gamma^{2}(\tau)} \frac{(\kappa-\ell)^{\tau-1}}{\left|(\kappa-\ell)^{\tau-1}\right|}\left(\frac{(\kappa-\hbar)^{\tau}}{\tau}\right)^{2}|\zeta(\ell)-v(\ell)|^{2} \\
& =\frac{1}{\Gamma^{2}(\tau+1)} \frac{(\kappa-\ell)^{\tau-1}(\kappa-\hbar)^{2 \tau}}{\left|(\kappa-\ell)^{\tau-1}\right|}|\varsigma(\ell)-v(\ell)|^{2} \\
& \leq \sigma \eta_{\beth}^{\nu}(\varsigma, v) .
\end{aligned}
$$

Thus, all the assumptions of Corollary 2.7 are verified, so $\partial$ has a unique fixed point, i.e., the Riemann-Liouville fractional integral equation has a unique solution.

\section{Existence of a unique solution of Atangana-Baleanu fractional operator}

In 2016, Atangana and Baleanu [41] developed more general definitions of a fractional derivative and an integral operator targeting nonlocal and nonsingular kernel. This operator takes the form (5.1). This study examines the connections between nanofluids, the dynamics of ions over the membrane, material mechanics, and predictor-corrector algorithms [42-46]. This new impression offers the opportunity to elaborate on the new findings/new insights and creative approaches for contextualizing the new topics in various aspects.

Let $\wp=C([0,1], \mathbb{R})$ be a set of all continuous functions from $[0,1]$ onto $\mathbb{R}$. Define the distance $\eta_{\beth}^{\nu}: \wp \times \wp \rightarrow[0, \infty)$ by

$$
\eta_{\beth}^{\nu}(\varsigma, v)=|\varsigma(\kappa)-v(\kappa)|^{2}
$$

for all $\varsigma(\kappa), v(\kappa) \in \wp$ and $\kappa \in[0,1]$. Also, define $], v: \wp \times \wp \rightarrow[1, \infty)$ by

$$
\begin{aligned}
& I(\varsigma(\kappa), v(\kappa))=\frac{|\zeta(\kappa)|+|v(\kappa)|}{2}+2, \\
& v(\varsigma(\kappa), v(\kappa))=\frac{|\zeta(\kappa)|+|v(\kappa)|}{1+|\zeta(\kappa)|+|v(\kappa)|}+2 .
\end{aligned}
$$

Then $\left(\wp, \eta_{\beth}^{\nu}\right)$ is an $\eta_{\beth}^{\nu}$-metric space.

Atangana-Baleanu fractional integral type (AB) of order $\Re$ of a function $\varsigma(\kappa)$ is exemplified as follows:

$$
{ }_{\hbar}^{\mathrm{AB}} I_{\kappa}^{\Re} \zeta(\kappa)=\frac{1-\Re}{\beta(\Re)} \zeta(\kappa)+\frac{\Re}{\beta(\Re) \Gamma(\Re)} \int_{\hbar}^{\kappa} \zeta(\ell)(\kappa-\ell)^{\Re-1} d \ell
$$

where $\Re \in(0,1], \varsigma(\kappa) \in \wp$ and $\kappa, \ell \in[0,1]$, which is the fractional integral. Note that the normalization functions $\beta(0)$ and $\beta(1)$ both are equal to 1 . 
Now, we will prove that the fractional integral (5.1) has a unique solution if the following assumption holds:

$$
\frac{1-\Re}{\beta(\Re)}+\frac{\hbar^{\Re}}{\beta(\Re) \Gamma(\Re)}<\sigma, \quad \text { where } \sigma \in(0,1) .
$$

Define an operator $\partial_{A B}: \wp \rightarrow \wp$ by

$$
\partial_{\mathrm{AB}} \zeta(\kappa)=\frac{1-\Re}{\beta(\Re)} \zeta(\kappa)+\frac{\Re}{\beta(\Re) \Gamma(\Re)} \int_{\hbar}^{\kappa} \zeta(\ell)(\kappa-\ell)^{\Re-1} d \ell .
$$

Thus, the solution of integral (5.1) is equivalent to finding a unique fixed point of the integral operator (5.2). Consider

$$
\begin{aligned}
& \eta_{\beth}^{\nu}(\partial \varsigma, \partial v)=|\partial \varsigma(\kappa)-\partial v(\kappa)|^{2} \\
& =\mid\left(\frac{1-\Re}{\beta(\Re)} \varsigma(\kappa)+\frac{\Re}{\beta(\Re) \Gamma(\Re)} \int_{\hbar}^{\kappa} \varsigma(\ell)(\kappa-\ell)^{\Re-1} d \ell\right) \\
& -\left.\left(\frac{1-\Re}{\beta(\Re)} v(\kappa)+\frac{\Re}{\beta(\Re) \Gamma(\Re)} \int_{\hbar}^{\kappa} v(\ell)(\kappa-\ell)^{\Re-1} d \ell\right)\right|^{2} \\
& =\left|\left(\frac{1-\Re}{\beta(\Re)}[\varsigma(\kappa)-v(\kappa)]+\frac{\Re}{\beta(\Re) \Gamma(\Re)} \int_{\hbar}^{\kappa}(\kappa-\ell)^{\Re-1} d \ell[\varsigma(\ell)-v(\ell)]\right)\right|^{2} \\
& \leq\left|\left(\frac{1-\Re}{\beta(\Re)}|\varsigma(\kappa)-v(\kappa)|+\left.\frac{\Re}{\beta(\Re) \Gamma(\Re)} \int_{\hbar}^{\kappa}(\kappa-\ell)^{\Re-1} d \ell\right|_{\zeta}(\ell)-v(\ell) \mid\right)\right|^{2} \\
& =\left|\left(\frac{1-\Re}{\beta(\Re)}|\zeta(\kappa)-v(\kappa)|-\frac{\Re}{\beta(\Re) \Gamma(\Re)}\left[\frac{(\kappa-\ell)^{\Re}}{\Re}\right]_{\hbar}^{\kappa}|\zeta(\ell)-v(\ell)|\right)\right|^{2} \\
& =\left|\left(\frac{1-\Re}{\beta(\Re)}|\varsigma(\kappa)-v(\kappa)|+\frac{\Re}{\beta(\Re) \Gamma(\Re)} \frac{(\kappa-\hbar)^{\Re}}{\Re}|\varsigma(\ell)-v(\ell)|\right)\right|^{2} \\
& \leq\left|\left(\frac{1-\Re}{\beta(\Re)}+\frac{\Re}{\beta(\Re) \Gamma(\Re)} \frac{(\kappa-\hbar)^{\Re}}{\Re}\right)\right| \varsigma(\kappa)-v(\kappa)||^{2} \\
& =\left(\frac{1-\Re}{\beta(\Re)}+\frac{\Re}{\beta(\Re) \Gamma(\Re)} \frac{(\kappa-\hbar)^{\Re}}{\Re}\right)^{2}|\zeta(\kappa)-v(\kappa)|^{2} \\
& \leq \sigma^{2}|\varsigma(\kappa)-v(\kappa)|^{2} \\
& \leq \sigma \eta_{\beth}^{\nu}(\varsigma, v) \text {. }
\end{aligned}
$$

Thus, the assertions managed by Corollary 2.7 are gratified, which implies that the fractional integral of Atangana-Baleanu type of order $\Re$ has a unique solution.

\section{Conclusion and discussions}

In this manuscript, we considered a double controlled metric space (in short, $\eta_{\beth}^{\nu}$-metric space). Via this space, some novel theoretical results involving fixed point techniques under various suitable assumptions have been established. To confirm our consequences, nontrivial examples have been presented. Finally, short and simple proofs have been obtained to find the existence and uniqueness of solutions of 2D Volterra integral equations, Riemann-Liouville integrals, and Atangana-Baleanu integral operators. In addition, the applications in this manuscript are listed as follows: 
- A fixed point technique to solve the 2D Volterra integral equation (3.1). This problem is considered without kernels because it is caused by a time-fractional telegraph equation. It is exciting to clarify a few points in this direction: In telegraph's equation characterizing the variation of voltage $\Lambda$ along with an electrical cable as a function of time and position

$$
\Lambda_{\kappa \kappa}+(\Phi+\Psi) \Lambda_{\kappa}+\Phi \Psi \Lambda=\complement^{2} \Lambda_{\zeta \zeta}
$$

which consists of a resistor of resistance $R$, a coil of inductance $L$, a resistor of conductance $\varkappa$, or a capacitor of capacitance $\complement$, where $\complement^{2}=\frac{1}{L L}, \Phi=\frac{\varkappa}{\complement}, \Psi=\frac{R}{L}$.

If $\frac{R}{L}=\frac{\varkappa}{\complement}$ (or $R \complement=L \varkappa$ ) a constant velocity of propagation would result and the attenuation would be minimized, this result was discussed by the physicist Oliver Heaviside in 1893.

This equation is a special case of the nonlinear Cauchy problem

$$
\frac{\partial^{2} \Lambda}{\partial \kappa^{2}}+\frac{\partial}{\partial \kappa}(q(\zeta, \kappa, \Lambda))=\frac{\partial^{2} \Lambda}{\partial \zeta^{2}}+\frac{\partial}{\partial \zeta}(q(\zeta, \kappa, \Lambda))+\Omega(\zeta, \kappa, \Lambda)
$$

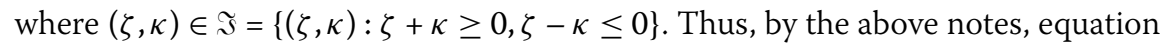
(6.1) can be written as the 2D Volterra integral equation (3.1).

- A fixed point technique to discuss the existence of a solution of Riemann-Liouville integral equations via Guo-Gupta-Suzuki-Cirić type results in the setting of an $\eta \underline{y}$-metric space.

- The existence and uniqueness of solutions for an Atangana-Baleanu fractional operator in the class of $\eta_{\beth}^{v}$-metric spaces have been discussed by a fixed point approach.

Acknowledgements

The second author would like to thank Sefako Makgatho Health Sciences University for funding this paper.

Funding

This research received no external funding.

Availability of data and materials

The data used to support the findings of this study are available from the corresponding author upon request.

Competing interests

The authors declare that they have no competing interests.

Authors' contributions

All authors contributed equally in writing this article. All authors read and approved the final manuscript.

\section{Author details}

'Department of Mathematics, Faculty of Science, Sohag University, Sohag 82524, Egypt. ${ }^{2}$ Institut Supérieur d'Informatique et des Techniques de Communication, Université de Sousse, H. Sousse, Tunisia. ${ }^{3}$ Department of Mathematics and Applied Mathematics, Sefako Makgatho Health Sciences University, Ga-Rankuwa, South Africa. ${ }^{4}$ China Medical University Hospital, China Medical University, Taichung 40402, Taiwan. ${ }^{5}$ Department of Mathematics and General Sciences, Prince Sultan University, Riyadh 11586, Saudi Arabia.

\section{Publisher's Note}

Springer Nature remains neutral with regard to jurisdictional claims in published maps and institutional affiliations. 


\section{References}

1. Amar, A.B., Jeribi, A., Mnif, M.: Some fixed point theorems and application to biological model. Numer. Funct. Anal. Optim. 29(1-2), 1-23 (2008)

2. Mc Ghee, D.F., Madbouly, N.M., Roach, G.F.: Applications of fixed point theorems to a chemical reactor problem. In: Integral Methods in Science and Engineering, pp. 133-138 (2004)

3. Border, K.C.: Fixed Point Theorems with Applications to Economics and Game Theory. Cambridge University Press, Cambridge (1985), 129 pages

4. Meznik, I.: Banach fixed point theorem and the stability of the market. In: Proceedings of the International Conference the Decidable and the Undecidable in Mathematics Education, Brno, Czech Republic, pp. 177-180 (2003)

5. Chwastek, K.: The applications of fixed-point theorem in optimisation problems. Arch. Electr. Eng. 61(2), 189-198 (2012)

6. Kakutani, S.: A generalization of Brouwer's fixed point theorem. Duke Math. J. 8(3), 457-459 (1941)

7. Agarwal, R.P., O'Regan, D., Wong, P.J.Y.: Positive Solutions of Differential, Difference and Integral Equations. Kluwer Academic, Dordrecht (1999)

8. Maleknejad, K., Torabi, P., Mollapourasl, R.: Fixed point method for solving nonlinear quadratic Volterra integral equations. Comput. Math. Appl. 62(6), 2555-2566 (2011)

9. Ahmad, B.: Existence of solutions for irregular boundary value problems of nonlinear fractional differential equations. Appl. Math. Lett. 23(4), 390-394 (2010)

10. Aydi, H., Jleli, M., Samet, B.: On positive solutions for a fractional thermostat model with a convex-concave source term via $\psi$-Caputo fractional derivative. Mediterr. J. Math. 17(1), 16 (2020)

11. Bai, Z:: On positive solutions of a nonlocal fractional boundary value problem. Nonlinear Anal. 72(2), 916-924 (2010)

12. Ameer, E., Aydi, H., Arshad, M., De la Sen, M.: Hybrid Ćirić type graphic $(\Upsilon, \Lambda)$-contraction mappings with applications to electric circuit and fractional differential equations. Symmetry 12(3), 467 (2020)

13. Agarwal, P., Baltaeva, U., Alikulov, Y.: Solvability of the boundary-value problem for a linear loaded integro-differential equation in an infinite three-dimensional domain. Chaos Solitons Fractals 140, 110108 (2020)

14. Agarwal, P., Denz, S., Jain, S., Alderremy, A.A., Aly, S.: A new analysis of a partial differential equation arising in biology and population genetics via semi analytical techniques. Phys. A, Stat. Mech. Appl. 542, 122769 (2020)

15. Khalid, A., Naeem, M.N., Agarwal, P., Ghaffar, A., Ullah, Z., Jain, S.: Numerical approximation for the solution of linear sixth order boundary value problems by cubic B-spline. Adv. Differ. Equ. 2019, 492 (2019)

16. Yassen, M.F., Attiya, A.A., Agarwal, P.: Subordination and superordination properties for certain family of analytic functions associated with Mittag-Leffler function. Symmetry 12(10), 1724 (2020)

17. Alderremy, A.A., Saad, K.M., Agarwal, P., Aly, S., Jain, S.: Certain new models of the multi space-fractional Gardner equation. Phys. A, Stat. Mech. Appl. 545, 123806 (2020)

18. Ameer, E., Aydi, H., Arshad, M., Alsamir, H., Noorani, M.S.: Hybrid multivalued type contraction mappings in $\alpha_{k}$-complete partial b-metric spaces and applications. Symmetry 11(1), 86 (2019)

19. Aydi, H., Lakzian, H., Mitrovic, Z.D., Radenovic, S.: Best proximity points of MF-cyclic contractions with property UC. Numer. Funct. Anal. Optim. 41(7), 871-882 (2020)

20. Hammad, H.A., De la Sen, M.: Tripled fixed point techniques for solving system of tripled-fractional differential equations. AIMS Math. 6(3), 2330-2343 (2020)

21. Kawasaki, T., Toyoda, M.: Fixed point theorem and fractional differential equations with multiple delays related with chaos neuron models. Appl. Math. 6, 2192-2198 (2015)

22. Lou, B.: Fixed points for operators in a space of continuous functions and applications. Proc. Am. Math. Soc. 127 1159-2264 (1999)

23. de Pascale, E., de Pascale, L.: Fixed points for some non-obviously contractive operators. Proc. Am. Math. Soc. 130 3249-3254 (2002)

24. Amann, H.: Fixed point equations and nonlinear eigenvalue problems in ordered Banach spaces. SIAM Rev. 18(4), 621-709 (1976)

25. Liu, J., Li, F., Lu, L.: Fixed point and applications of mixed monotone operator with super linear non-linearity. Acta Math. Sci. Ser. A 23(1), 19-24 (2003)

26. Abdeljawad, T., Agarwal, R.P., Karapinar, E., Kumari, P.S.: Solutions of the nonlinear integral equation and fractional differential equation using the technique of a fixed point with a numerical experiment in extended b-metric space. Symmetry 11, 686 (2019)

27. Hammad, H.A., De la Sen, M.: A coupled fixed point technique for solving coupled systems of functional and nonlinear integral equations. Mathematics 7, 634 (2019)

28. Hammad, H.A., De la Sen, M.: A solution of Fredholm integral equation by using the cyclic $\eta_{s}^{q}$-rational contractive mappings technique in b-metric-like spaces. Symmetry 11, 1184 (2019)

29. Karapinar, E., Czerwik, S., Aydi, H.: $(\alpha, \psi)$-Meir-Keeler contraction mappings in generalized b-metric spaces. J. Funct. Spaces 2018, Article ID 3264620 (2018)

30. Zhai, C.B., Hao, M.R.: Fixed point theorems for mixed monotone operators with perturbation and applications to fractional differential equation boundary value problems. Nonlinear Anal. 75, 2542-2551 (2012)

31. Kilbas, A.A., Srivastava, H.M., Trujillo, J.J.: Theory and Applications of Fractional Differential Equations. Elsevier Amsterdam (2006)

32. Kamran, T., Samreen, M., UI Ain, Q.: A generalization of $b$-metric space and some fixed point theorems. Mathematics 5, $19(2017)$

33. Mlaiki, N., Aydi, H., Souayah, N., Abdeljawad, T:: Controlled metric type spaces and the related contraction principle. Mathematics 6(10), 194 (2018)

34. Abdeljawad, T., Mlaiki, N., Aydi, H., Souayah, N.: Double controlled metric type spaces and some fixed point results. Mathematics 6, 320 (2018)

35. Bakhtin, I.A.: The contraction mapping principle in almost metric spaces. Funct. Anal. 30, 26-37 (1989)

36. Czerwik, S.: Contraction mappings in b-metric spaces. Acta Math. Inform. Univ. Ostrav. 1, 5-11 (1993)

37. Mckee, S., Tang, T., Diogo, T.: An Euler-type method for two-dimensional Volterra integral equations of the first kind. IMA J. Numer. Anal. 20, 423-440 (2000) 
38. Dobner, H.J.: Bounds for the solution of hyperbolic problems. Computing 38, 209-218 (1987)

39. Xie, W., Xiao, J., Luo, Z.: Existence of solutions for Riemann-Liouville fractional boundary value problem. Abstr. Appl. Anal. 2014, Article ID 540351 (2014)

40. Eloe, P.W., Jonnalagadda, J.: Quasilinearization and boundary value problems for Riemann-Liouville fractional differential equations. Electron. J. Differ. Equ. 2019, 58 (2019)

41. Atangana, A., Baleanu, D.: New fractional derivatives with nonlocal and non-singular kernel: theory and application to heat transfer model. Therm. Sci. 20(2), 763-769 (2016)

42. Saqib, M., Khan, I., Shafie, S.: Application of Atangana-Baleanu fractional derivative to MHD channel flow of CMC-based CNT's nanofluid through a porous medium. Chaos Solitons Fractals 116, 79-85 (2018)

43. Bahaa, G.M.: Optimal control problem for variable-order fractional differential systems with time delay involving Atangana-Baleanu derivatives. Chaos Solitons Fractals 122, 129-142 (2019)

44. Atangana, A., Koca, I.: Chaos in a simple nonlinear system with Atangana-Baleanu derivatives with fractional order. Chaos Solitons Fractals 89, 447-454 (2016)

45. Owolabi, K.M., Atangana, A.: On the formulation of Adams-Bashforth scheme with Atangana-Baleanu-Caputo fractional derivative to model chaotic problems. Chaos, Interdiscip. J. Nonlinear Sci. 29(2), 023111 (2019)

46. Gomez-Aguilar, J.F., Atangana, A., Morales-Delgado, V.V.F.: Electrical circuits RC, LC, and RL described by Atangana-Baleanu fractional derivatives. Int. J. Circuit Theory Appl. 45(11), 1514-1533 (2017)

\section{Submit your manuscript to a SpringerOpen ${ }^{\circ}$ journal and benefit from:}

- Convenient online submission

- Rigorous peer review

- Open access: articles freely available online

- High visibility within the field

- Retaining the copyright to your article

Submit your next manuscript at $>$ springeropen.com 